\title{
Applying the Bi-level HMM for Robust Voice-activity Detection
}

\author{
Yongwon Hwang*, Mun-Ho Jeong ${ }^{\dagger}$, Sang-Rok Oh** and Il-Hwan Kim***
}

\begin{abstract}
This paper presents a voice-activity detection (VAD) method for sound sequences with various SNRs. For real-time VAD applications, it is inadequate to employ a post-processing for the removal of burst clippings from the VAD output decision. To tackle this problem, building on the bilevel hidden Markov model, for which a state layer is inserted into a typical hidden Markov model (HMM), we formulated a robust method for VAD not requiring any additional post-processing. In the method, a forward-inference-ratio test was devised to detect the speech endpoints and Mel-frequency cepstral coefficients (MFCC) were used as the features. Our experiment results show that, regarding different SNRs, the performance of the proposed approach is more outstanding than those of the conventional methods.
\end{abstract}

Keywords: Voice-activity detection, Bi-level HMM, Forward-inference-ratio test

\section{Introduction}

Voice-activity detection (VAD) has become increasingly important since the recent application of speech-recognition technology in the design of smart devices, electric home appliances, and the vocal interfaces of cars, among others [1]. The main purpose of a VAD system is the correct identification of the presence of either speech frames or the separate speech and non-speech patterns of a given signal. In the current context, VAD has been treated as a preprocessing technology for plentiful voice-related algorithms such as voice query, effective voice coding under a limited bandwidth, speaker recognition, and speech enhancement [1-4].

A key difficulty regarding VAD is the identification of speech endpoints in the presence of non-stationary noisy signals. Recently, a number of approaches based on statistical models have been developed [7-14], and the corresponding performances in noisy environments are more favorable than those of early methods for which only magic numbers or a portion of rules are used $[5,6]$. Typical examples of these newer approaches are those for which either the likelihood-ratio test (LRT) [7, 14], the hidden Markov model (HMM) [10-12], or the support vector machine (SVM) are used [15]. However, these approaches are still problematic because burst clippings, the short frames of false positives and false negatives, are often caused. The burst clippings can be removed, however, by applying rulebased post-processing to the output decisions of these

$\dagger \quad$ Corresponding Author: School of Robotics, Kwangwoon University, Korea. (mhjeong@kw.ac.kr)

* Dept. of Electrical and Electronic Engineering, Yonsei University, Korea. (nicecogbot@gmail.com)

* Center for Robotics Research, Korea Institute of Science and Technology, Korea. (sroh@kist.re.kr)

*** Dept. of Electronic and Communication, Kwangwoon National University, Korea. (ihkim@kangwon.ac.kr)

Received: January 16, 2016; Accepted: May 29, 2016 methods; but, although rule-based post-processing is simple, it is unreliable in a non-stationary, noisy environment and is insufficient for real-time applications. To eliminate burst clippings, Sohn [16] devised a HMM-based hangover method for which the state-duration effect of the HMM is utilized. However, its limitation is that a little success in avoiding burst clippings relies on the HMM whose weakness is in modeling state duration [17]. The heuristic hangover mechanism introduced by Veisi [10], whereby the short-period states that are decided by the likelihood ratio are ignored, shows a favorable performance, but it is problematic in that the lengths of the short-period states should be adequately determined adequately against variable noises. Tiaw and Jeong [17] suggested a bi-level HMM for which a state layer is inserted into a typical HMM, and applied it to visual VAD. They contributed a stochastic model for which neither postprocessing nor any rule-based method for the elimination of the burst clippings on the decided state sequences are required.

In this paper, we present our formulation of the bi-level HMM for the VAD problem. Mel-frequency cepstral coefficients (MFCC) are used as the features that constitute the bi-level-HMM observation layer. The states that correspond to the features' probabilistic density functions form the lower layer of the bi-level HMM, while the upper layer is composed of the states that represent whether speaking is performed or not. We also introduce the forward inference ratio test that is effective to alleviate burst clippings. To evaluate the proposed method, we compared it with the methods based on the Gaussian mixture model (GMM) and HMM. The experiments that we conducted under different SNRs show that our approach is more efficient than the conventional methods.

\section{MFCC Features}

MFCC features have been widely used in the field of 
sound processing. The mel-frequency cepstrum is a representation of the short-term power spectrum of a sound, and is based on the discrete cosine transform of a logpower spectrum that is on the nonlinear mel-scale of a frequency. A distinction between the mel-frequency cepstrum and the cepstrum is that the mel-frequency bands are equally spaced on the mel-scale that approximates the response of the human auditory system [18]. The MFCC is obtained from the following processes [18]:

(1) High-pass filtering of a voice signal to compensate for the suppressed high-frequency parts of the human sound system.

(2) Frame-blocking to create an observation unit composed of $N$ adjacent voice samples, whereby voice signals are sampled at $8 \mathrm{khz}$ and constitute one frame with 200 samples ( $25 \mathrm{~ms}) ; 80$ samples $(10 \mathrm{~ms})$ are overlapped between two adjacent frames.

(3) Applying a Hamming window to increase the continuity of two adjacent frames:

$$
\begin{aligned}
& s^{\prime}(n)=s(n) \cdot w(n) \\
& w(n)=0.54-0.4 \cos [2 \pi n /(N-1)], 0 \leq n \leq N-1
\end{aligned}
$$

where $s(n)$ is a voice signal sample.

(4) Fast Fourier Transform (FFT) to transform the timedomain signal into a frequency

(5) Mel-filtering where $M$ triangle band-pass filters are applied to the FFT spectrum, followed by the computation of the logarithmic energy of each filter output.

(6) Discrete cosine transform (DCT) on the Mel-filtered output to acquire 13 coefficients.

Among the thirteen coefficients, the first coefficient corresponds to the short-term energy and does not help with the discrimination of the voice signal in the case of severe noises. The coefficients from the tenth to the thirteenth are influenced by the artifacts of a numerical analysis such as the Hamming window [19]. Eight coefficients are therefore selected from the second to the ninth as features for VAD.

\section{Voice-activity Modeling with Bi-level HMM}

\subsection{Probabilistic sound states}

It is necessary to divide sampled sound signals into speech and non-speech intervals for the plentiful voicerelated algorithms such as voice query, effective voice coding under a limited bandwidth, speaker recognition, and speech enhancement. A bottleneck issue, however, is the impurity of the speech and non-speech intervals; that is, non-speech features are often found in speech intervals due to voiceless sounds or the existence of inter-word gaps, and because of non-stationary noises, speech features can also

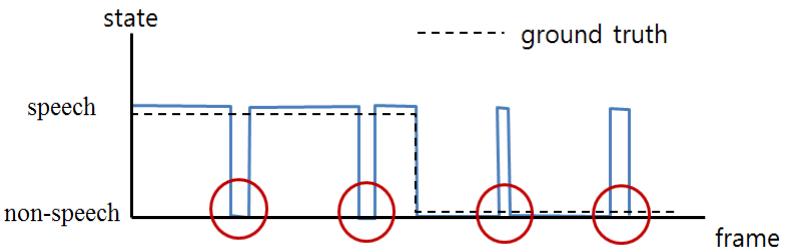

Fig. 1. Burst Clipings

exist in non-speech intervals. These bottleneck features can also cause the burst clippings that are the short frames of false positives and false negatives, that is, wrong decisions of states in a non-speech interval or a speech interval as shown in Fig. 1.

Two assumptions, for which the idea in [17] serves as the basis for the probabilistic modeling of sound states, have therefore been formulated to address this bottleneck difficulty. According to the first assumption, two sound signal states for activeness such as active and inactive exist, allowing for the stochastic definition of the MFCC feature vectors, as shown in (2). For the other assumption, two speech states such as speech and non-speech are acknowledged in sound signals that are stochastically determined by the frequencies of the active and inactive states, as shown in (3); accordingly, the active states occur more frequently than the inactive ones in the speech state, whereas the inactive states are dominant in the non-speech state. The state definition according to these assumptions is somewhat relaxed in that inactive states are allowed in the speech state, but in the next section, the definition plays a key role in the removal of the burst clippings during the decoding of the speech states in the bi-level HMM.

$$
\begin{gathered}
q_{t}^{A} \in\{\text { active, inactive }\}: \\
p\left(o_{t} \mid q_{t}^{A}\right)=\sum_{k=1}^{K} w_{k} G\left(o_{t}, \mu_{k}^{A}, \Pi_{k}^{A}\right), \sum_{k=1}^{K} w_{k}=1 \\
q_{t}^{S} \in\{\text { speech, nonspeech }\}: \\
p\left(q_{t}^{S} \mid q_{t}^{S}\right)=B_{q_{t}^{S} q_{t}^{A}}, \sum_{A} B_{q_{t}^{S} q_{t}^{A}}=1
\end{gathered}
$$

where $o_{t}$ is an eight-dimensional MFCC feature vector at time $t, G\left(o_{t}, \mu_{k}^{A}, \Pi_{k}^{A}\right)$ is the $k_{\mathrm{th}}$ Gaussian density function of $o_{t}$ with the mean vector $\mu_{k}^{A}$ and the covariance matrix $\Pi_{k}^{A}$, and $B$ is the frequency matrix whose element represents a frequency of active or inactive in speech or non-speech.

\subsection{Bi-level HMM for VAD}

The bi-level HMM can be considered an extension of the typical HMM, as it comprises an intermediate state layer between the discrete state layer and the observation layer as shown in Fig. 2. By applying the probabilistic sound states to the bi-level HMM, the speech states correspond to the upper-state layer, taking on the $1^{\text {st }}$-order Markov 


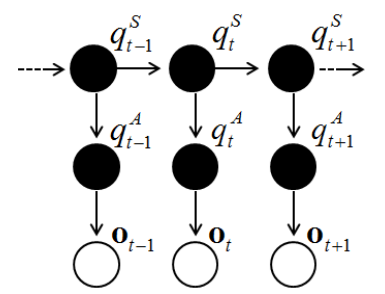

Fig. 2. Bi-level HMM for VAD

property, and depending on the states in the upper-state layer, the activeness states correspond to the lower-state layer. The MFCC feature vectors are considered observations in the observation layer.

\subsection{Forward inference ratio test}

The forward inferences of the speech states are performed recursively [17], as follows:

$$
\begin{aligned}
\alpha_{t}\left(j^{S}\right) & =p\left(o_{1}, \cdots, o_{t}, q_{t}^{S}=j^{S} \mid \Theta\right) \\
& =\sum_{j^{A}=\text { active }} b_{j^{S} j^{A}} p_{j^{A}}\left(o_{t}\right) \sum_{i^{S}=\text { speech }} a_{i^{S}} \alpha_{j^{S}}\left(i^{S}\right)
\end{aligned}
$$

where $\Theta$ represents the parameters of the bi-level HMM for VAD and includes the state-transition probabilities, $a_{i^{S} j^{S}}=p\left(q_{t}^{S}=j^{S} \mid q_{t-1}^{S}=i^{S}\right)$, the conditional probabilities of the active states, $b_{j^{S} j^{A}}=p\left(q_{t}^{A}=j^{A} \mid q_{t}^{S}=j^{S}\right)$, and the observation distribution, $p_{j^{A}}\left(o_{t}\right)=p\left(o_{t} \mid q_{t}^{A}=j^{A}\right)$. The difference between (4) and the HMM forward inference is the insertion of $b_{j^{S} j^{A}}$. This difference helped in the suppression of the undesirable state changes that are caused by noisy signals.

$$
\begin{aligned}
& p\left(q_{t}^{s} \mid o_{1}, \cdots, o_{t}, \Theta\right) \\
& =\frac{p\left(o_{1}, \cdots, o_{t}, q_{t}^{s} \mid \Theta\right)}{p\left(o_{1}, \cdots, o_{t} \mid \Theta\right)} \propto p\left(o_{1}, \cdots, o_{t}, q_{t}^{s} \mid \Theta\right) \\
& q_{t}^{s}=\left\{\begin{array}{l}
\text { speech if } \alpha_{t}\left(j^{s}=\text { speech }\right)>\alpha_{t}\left(j^{s}=\text { nospeech }\right) \\
\text { nospeech otherwise }
\end{array}\right.
\end{aligned}
$$

According to (5), the posterior probability at time $t$ is proportional to the forward inference, so the speech states can be decided by a comparison between the corresponding forward inferences, like those in (6). However, in order to alleviate the burst clippings more, the forward-inferenceratio test as defined in (7) is used to recognize the speech states:

$$
q_{t}^{s}= \begin{cases}\text { speech } & \text { if } \frac{\alpha_{t}\left(j^{S}=\text { speech }\right)}{\alpha_{t}\left(j^{S}=\text { nospeech }\right)}>\xi \\ \text { nospeech } & \text { otherwise }\end{cases}
$$

where $\xi$ is a threshold.

\section{Experiment Results}

NOIZEOUS is an open speech database wherein the sound sequences are composed of successive words and recorded in various environments with a variety of noise levels [20]. For the experiment we used the sound sequences in the four environments such as car, restaurant, street and train. The proposed method was compared with the methods based on GMM and HMM. With respect to each environment, 10 audio files were used as the training samples for the learning stage and another 10 audio files were randomly selected for the test stage. Post-processing was not applied for the removal of the burst clippings.

Fig. 3 shows the results of the speech-state estimation according to the SNRs for the car environment. As the energies of the inter-word short voiceless intervals are generally low, these intervals tend to be regarded as the non-speech state. Such tendency is most pronounced in the case that the GMM-based method is applied. The HMMbased method shows better results than the GMM because of the state-duration effect of HMM. Frequent burst clippings found in both the GMM and the HMM cases can lead to a poor performance regarding real-time voicerelated algorithms such as voice coding under a limited bandwidth and speech recognition. The performance of the bi-level-HMM-based VAD is more outstanding than those of the other conventional approaches. It reveals that the proposed method suppresses burst clippings effectively.

As shown in (8), the speech-recognition rate $\left(P_{D}\right)$ and the state-change false rate $\left(P_{S C}\right)$ were used for the evaluation

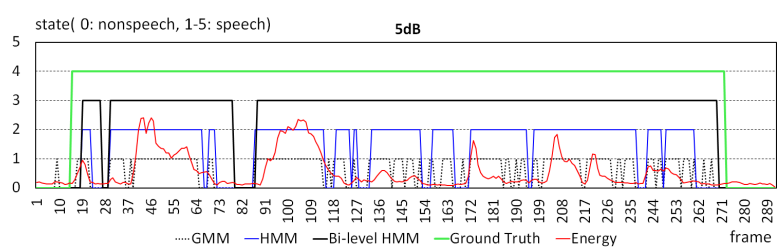

(a)

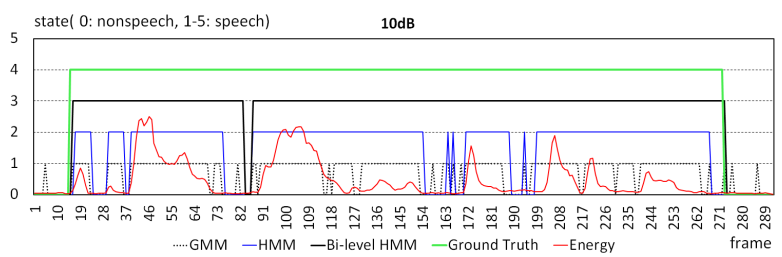

(b)

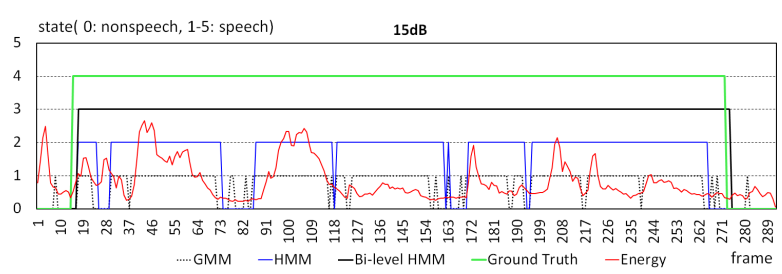

(c)

Fig. 3. VAD results: (a) $5 \mathrm{~dB}$; (b) $10 \mathrm{~dB}$; (c) $15 \mathrm{~dB}$ 
of the proposed approach. Fig. 4 shows the performance comparison for which the ROC (Receiving Operating Characteristic) space wa used, whereby it is proven that the proposed approach outperforms the conventional methods.

$$
\begin{gathered}
P_{D}=\frac{\text { No. of frames recognized as speech state }}{\text { No. of frames of ground truth }} \\
P_{S C}=\frac{1}{2}\left(\begin{array}{l}
\text { No. of transitions from speech to } \\
\frac{\text { nonspeech in speech interval }}{\text { No. of frames of speech intervals }} \\
\begin{array}{l}
\text { No. of transitions from nonspeech to } \\
\frac{\text { speech in nonspeech interval }}{\text { No. of frames of nonspeech intervals }}
\end{array}
\end{array}\right)
\end{gathered}
$$

\section{Conclusion}

In this paper, we present a VAD method for which the bi-level HMM is employed. For most of the conventional VAD methods, additional post-processing such as rulebased delayed frames and hangover mechanisms must be employed to remove the burst clippings from the VAD output decision; however, this is undesirable for real-time VAD applications. So that consecutive speech frames could be detected without any post-processing, we applied the bilevel HMM to VAD and devised a forward-inference-ratio test to determine the speech frames. Our experiments then proved that the presented method can be used successfully to recognize speech intervals with few burst clippings without any post-processing.

\section{Acknowledgements}

This work is supported by the Research Grant of Kwangwoon University in 2013.

\section{References}

[1] Y. Zhang, Z. Tang and Y. Li, et al., "A hierarchical framework approach for voice activity detection and speech enhancement", The Scientific World J., Vol. 2014, pp. 1-8, 2014.

[2] J. Choi, "Speech and Noise Recognition System by Neural Network", The J. of Korea Institute of Electronic Communication Science, Vol. 5, No. 4, pp. 357-362, 2010.

[3] J. Choi, "Subband Based Spectrum Subtraction Algorithm", The J. of Korea Institute of Electronic Communication Science, Vol. 8, No. 4, pp. 555-560, 2013.

[4] C. Lee and D. Kim, "Adaptive Noise Reduction of
Speech Using Wavelet Transform", The J. of Korea Institute of Electronic Communication Science, Vol. 4, No. 3, pp. 190-196, 2009.

[5] M. H. Moattar , M. M. Homayounpour, "A Simple but Efficient Real-Time Voice Activity Detection Algorithm", European Signal Processing Conference, pp. 2549-2553, 2009.

[6] R. V. Prasad, A. Sangwan, H. S. Jamadagni, Chiranth M. C, Rahul Sah, Vishal Gaurav, "Comparison of Voice Activity Detection Algorithms for VoIP", Proc. of the 7th International Symposium on Computers and Communications, pp. 1530-1346, 2002.

[7] J. Ramirez, J. C. Segura, C. Benitez, L. Garcia, and A. Rubio, "Statistical Voice Detection using a Multiple Observation Likelihood Ratio Test", IEEE Signal Proc. Letters, Vol. 12, No. 10, pp. 689-692, 2005.

[8] Joon-Hyuk Chang , Nam Soo Kim, S. K. Mitra, "Voice activity detection based on multiple statistical models", IEEE Trans. on Signal Processing, Vol. 54, Issue 6, 1965-1976, 2006.

[9] Ji Wu, Xiao-Lei Zhang, “An efficient voice activity detection algorithm by combining statistical model and energy detection", EURASIP Journal on Advances in Signal Processing, Vol. 2011, No. 18, 2011.

[10] H. Veisi and H. Sameti, "Hidden Markov Modelbased Voice Activity Detector with High Speech Detection Rate for Speech Enhancement", IET Signal Proc., Vol. 6, No. 1, pp. 54-63, 2012.

[11] H. Othman and T. Aboulnasr, "A Semi-Continuous State-Transition Probability HMM-Based Voice Activity Detector", EURASIP J. on Audio, Speech, and Music Proc., Vol. 2007, pp. 1-7, 2007.

[12] Yuan Liang, Xianglong Liu1, Yihua Lou, Baosong Shan, "An improved noise-robust voice activity detector based on hidden semi-Markov models", Pattern Recognition Letters, Vol. 32, pp. 1044-1053, 2011.

[13] Xulei Bao, Jie Zhu, "A Novel Voice Activity Detection based on Phoneme Recognition Statistical Model", EURASIP Journal on Audio, Speech, and Music Processing, Vol. 2012, No. 1(doi:10.1186/ 1687-4722-2012-1), 2012.

[14] D. A. Reynolds, T. F. Quatieri, and R. B. Dunn, "Speaker Verification using Adapted Gaussian Mixture Models", Digital Signal Processing, Vol. 10, pp. 1941, 2000.

[15] S. Chen, R. C. Guido, T. Truong, and Y. Chang, "Improved Voice Activity Detection Algorithm using Wavelet and Support Vector Machine", Computer Speech and Language, Vol. 24, No. 3, pp. 531-543, 2010.

[16] J. Sohn, N.-S. Kim, and W. Sung, "A statistical model-based voice activity detection", IEEE Signal Proc. Letters, Vol. 6, No. 1, pp. 1-3, 1999.

[17] P. Tiawongsombat, Mun-Ho Jeong, J. Yun, B. You, and S. Oh, "Robust visual speakingness detection 
using bi-level HMM", Pattern Recognition, Vol. 45, No. 2, pp. 783-793, 2012.

[18] H. Wang, Y. Xu, and M. Li, "Study on the MFCC similarity-based voice activity detection algorithm, Int. Conf. on Artificial Intelligence", Management Science and Electronic Commerce(AIMSEC), Deng Leng, pp. 4391-4394, August 2011.

[19] S. Skorik and F. Berthommier, "On a cepstrum-based speech detector robust to white noise", Computing Research Repository, Vol. cs.CL/00100014, pp. 1-4, 2000.

[20] NOIZEUS: A noisy speech corpus for evaluation of speech enhancement algorithms. Available online: http://ecs.utdallas.edu/loizou/speech/noizeus.

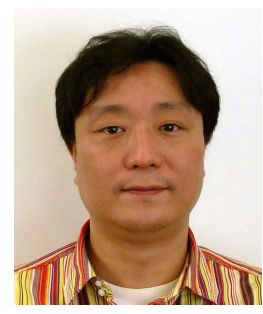

Yongwon Hwang received the M.S. degrees from dept. of electronic engineering for control and biomedical, Konkuk University and he is doing Ph.D. from the dept. of electronics engineering for intelligence control system, Yonsei University, Korea. He has been a researcher at Korea institute of science and technology since 2002. His major interests are robot vision, intelligence, cognitive science, and humanrobot interaction.

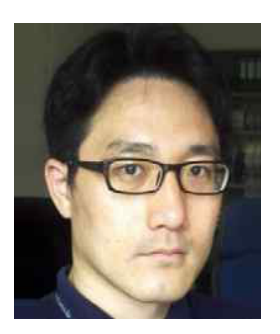

Mun-Ho Jeong received the B.S. and M.S. degrees from Korea advanced institute and science and technology (KAIST), Korea and his Ph.D. from the dept. of mechanical engineering for computer controlled systems, Osaka University, Japan. He has been a professor at the school of robotics in Kwangwoon University since 2010. His major interests are robot vision and intelligence, image processing, and human-robot interaction.

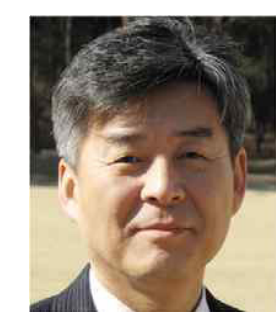

Sang-Rok Oh joined KIST in 1988 after graduation from KAIST for his $\mathrm{Ph} . \mathrm{D}$. degree, and has been working as a principal research engineer at the center for robotics research, KIST. His research interests include information and communication technology, biorobotics for quality of life, and network based intelligent service robots.

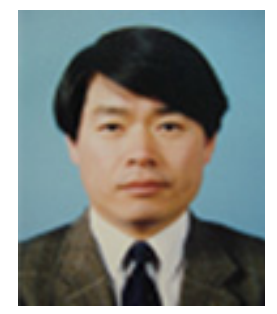

Il-Hwan Kim received B.S. and M.S. degree in control and instrument engineering from Seoul national university in 1982 and 1985 respectively and $\mathrm{Ph} . \mathrm{D}$. at the Tohoku university in 1993. In 1995, he joined the dept. of electrical and electronic engineering at the Kangwon national university and is currently a professor. His research interests include control, mechatronics, and human interfaces. 